\title{
Los significados del bachillerato para los jóvenes y la permanencia escolar en México
}

\section{The meanings of high school for young people and student permanency in Mexico}

\section{EDUARDO WEISS HORZ*}

Este artículo discute los lineamientos de la política de la educación media superior derivados de su obligatoriedad a la luz de hallazgos de estudios sobre el significado del bachillerato para los jóvenes y las razones del abandono escolar. Los estudios empíricos de referencia provienen de la línea de investigación cualitativa del autor desde una perspectiva sociocultural y de las tesis de maestría y doctorado dirigidas en la línea de investigación sobre Jóvenes y bachillerato, y se ponen en diálogo con los lineamientos en documentos oficiales. Para los jóvenes, la escuela significa un espacio de vida juvenil que resta tiempo a las actividades académicas a la vez que permite importantes procesos de subjetivación; entre las razones del abandono, destacan la reprobación y la falta de interés en los estudios. Hay una brecha entre las escasas competencias con las que llegan gran parte de los estudiantes al bachillerato y las exigencias académico-propedéuticas del plan y programas de estudios. Por la flexibilización de la acreditación sin cambios en los planes de estudio, el bachillerato corre el peligro de convertirse en una guardería de los jóvenes en vez de fomentar competencias para la vida.

This essay discusses the policy guidelines for compulsory high school studies in light of research findings on the meaning of high school for young people and the reasons for their withdrawal. The empirical studies of reference are from the author's qualitative research, from a sociocultural perspective, and from the direction of master's and doctoral theses on young people and high school; these findings are compared with the guidelines contained in official documents. It is shown that for young people, school means-among other things-a space for youthful life that reduces the time for academic activities while permitting important processes of subjectivization. The main reasons for withdrawal are academic failure and disinterest in academic subjects. A gap exists between the low skills of most entering high school students, and the academic demands of the plan and program of studies. Due to the increased flexibility of earning course credit without changes in plans of study, high school runs the risk of becoming a nursery for young people, instead of a place for learning life skills.

\section{Palabras clave:}

bachillerato, permanencia, jóvenes, México

\section{Keywords:}

high school, retention, young people,

Mexico

Recibido: 27 de febrero de 2018. Aceptado para su publicación: 22 de junio de 2018. Recuperado de: https://sinectica.iteso.mx/index.php/SINECTICA/article/view/856 DOI: $10.31391 / \mathrm{S} 2007-7033(2018) 0051-003$

*Doctor en Ciencias Sociales por la Universidad Erlangem de Alemania. Profesor-investigador del Centro de Investigación y de Estudios Avanzados (Cinvestav) del Instituto Politécnico Nacional. Sus líneas de investigación son procesos de formación en educación secundaria o educación media superior. Correo electrónico: eweiss@cinvestav.mx 


\section{INTRODUCCIón}

La política de ampliación de la cobertura y disminución del abandono escolar

E n las últimas décadas, la educación media superior mexicana ha crecido de manera continua por encima del aumento poblacional: pasó de 2.1 millones de alumnos en 1990-1991 a 4.4 millones en 2012-2013. Aun así, México era de los países con más rezago en la cobertura de la educación media superior. Según datos del Sistema de Información de Tendencias Educativas en América Latina (SITEAL, 2015), la tasa de escolarización de la población de quince a diecisiete años de edad en México pasó de 57.9\% en 2000 a 69.6\% en 2013 (frente a 88\% en Argentina, el país con la tasa más alta en América Latina, o 75.5\% en Brasil, un país con tasas por lo general comparables con las de México). Los organismos internacionales y los gobiernos vigilan celosamente los avances en las tasas de cobertura y la disminución de las tasas de abandono escolar.

En este contexto, en México se agregó al artículo $3^{\circ}$ de la Constitución, en 2012, la obligatoriedad de la educación media superior. El artículo transitorio especifica la obligación del Estado -federación, estados y municipios- de ofrecer un lugar para cursar la educación media superior a quien, teniendo la edad típica, hubiera concluido la educación básica. Se planteó concretar el cumplimiento de la obligación de manera gradual y creciente a partir del ciclo escolar 2012-2013 hasta lograr la cobertura total en sus diversas modalidades en el país a más tardar en el ciclo escolar 2021-2022. Un año después, una nueva reforma al artículo $3^{\circ}$ incluyó, entre otros cambios, que el Estado garantizara la calidad en la educación obligatoria. Asimismo, el artículo 31 de la Constitución establece la obligación de los mexicanos de fomentar en sus hijos o pupilos la asistencia a la escuela en todos los niveles considerados como obligatorios (Diario Oficial de la Federación, 2012).

En el Programa Sectorial de Educación 2013-2018, el gobierno se fijó la meta ambiciosa de aumentar la cobertura de la educación media superior de $65.9 \%$ (ciclo 20122013) a $80 \%$ en 2018. Entre los ciclos 2012-2013 y 2016-2017, la matrícula total de la educación media superior pasó de 4.44 a 5.48 millones de alumnos, es decir, observó un aumento de más de un millón de estudiantes, lo que significa un crecimiento de $23.4 \%$. La matrícula escolarizada se elevó en casi setecientos mil estudiantes entre 2012-2013 y 2016-2017 y la no escolarizada, en 360,000 estudiantes. De esta manera se logró una cobertura escolarizada de $76.6 \%$ y, si se considera la no escolarizada, de $82.0 \%$ (Consejo Nacional de Autoridades Educativas. Educación Media Superior [Conaedu], 2017).

Según datos del Instituto Nacional para la Evaluación de la Educación (INEE, 2016), la tasa de asistencia de jóvenes de quince a diecisiete años era de $73.2 \%$ en 2015, mayor en el caso de mujeres (73.7\%) que en el de los hombres (72.7\%). Los jóvenes rurales asistían en $62.2 \%$, los indígenas, en 63.9\%, y los hablantes de lenguas indígenas, solo en $56.7 \%$.

Medidas importantes para lograr el objetivo de la ampliación de cobertura fueron el fondo de inversión para ampliar la infraestructura y el de ampliación de la cobertura, y también nuevos tipos de servicio, como el telebachillerato comunitario y la "prepa en línea", a la vez que se ampliaba la "prepa abierta" (Conaedu, 2015).

Un ingrediente muy importante para lograr la ampliación de la cobertura y disminuir el abandono escolar han sido las becas, tanto las del programa Prospera (antes 
Oportunidades) dirigidas a localidades de pobreza y marginación social, que otorga la Secretaría de Desarrollo Social, como las del Programa de Becas de la Educación Media Superior, que incluyó, además, una nueva modalidad: la beca contra la deserción que se ofrece de manera más flexible (Weiss, 2015a). En el nivel medio superior, el programa de becas de la educación media superior apoyó a 731,537 estudiantes; por su parte, Prospera brindó 1,365,848 apoyos. Un total de 2,086,412 de alumnos de educación media superior, cuyo ingreso familiar se encuentra en los primeros cuatro deciles, cuentan con algún apoyo, lo que significa una cobertura de 87\% (Conaedu 2017).

Un pilar importante de la ampliación de la cobertura sería la reducción del abandono escolar: "cerrar la llave de la deserción", como se decía entonces de manera coloquial. Históricamente, la tasa de deserción rondaba alrededor de 16.5\%; el esfuerzo fue importante en el sexenio anterior de 2007-2012; se logró reducir la tasa a 14.3\% en 2012-2013 mediante la ampliación del número de becas y el programa Síguele, que promovía tutorías académicas, el fortalecimiento de habilidades socioemocionales (Construye-T) y un sistema centralizado de alerta temprana sobre alumnos en riesgo de abandono. El Programa Sectorial de Educación 2013-2018 se propuso reducirla por un tercio de 14.3\% en 2012 a 9\% en 2018 (Conaedu, 2015), una meta que se veía excesiva para cualquier sistema educativo en tan corto tiempo.

Los estudios sobre el abandono escolar revelan que sus causas son multifactoriales (Székely, 2015; Weiss, 2015a; INEE, 2017a). Uno de los más representativos es la Encuesta Nacional de Educación Media Superior (ENDEMS, 2011) aplicada por la Secretaría de Educación Pública y el Consejo para la Evaluación de la Educación del Tipo Medio Superior (SEP-COPEMS, 2012) a una muestra representativa a nivel nacional: 13,014 jóvenes $(4,779$ que nunca se matricularon en el nivel medio superior; 2,549 que desertaron y 5,686 no desertores que lo concluyeron o que continúan estudiando). En la tabla 1 agrupamos las 23 razones manifiestas y más frecuentes para abandonar la escuela en tres grandes categorías: económicas, académicas o escolares y personales o familiares.

Tabla 1. Razones de abandono escolar de los "desertores"

\begin{tabular}{|c|c|c|}
\hline Razones económicas & Razones académicas y escolares & Razones personales o familiares \\
\hline \multirow{7}{*}{$\begin{array}{l}\text { 36.4\%: "Faltaba dinero } \\
\text { en el hogar" } \\
\text { 7.2\%: "Consideraba } \\
\text { trabajar más importante } \\
\text { que estudiar" }\end{array}$} & \multirow{3}{*}{$\begin{array}{c}\text { 7.8\%: "Le disgustaba estudiar" } \\
\text { 6.0\%: "Le dieron de baja por reprobar } \\
\text { materias" }\end{array}$} & \multirow{4}{*}{$\begin{array}{c}\text { 1.1\%: "Falleció un familiar" } \\
\text { 1.7\%: "Problemas con sus padres" } \\
\text { 1.0\%: "Se cambió de casa" } \\
\text { 0.9\%: "Baja autoestima" }\end{array}$} \\
\hline & & \\
\hline & & \\
\hline & $\begin{array}{c}\text { 7.1\%: "Problemas para entender a los } \\
\text { maestros" }\end{array}$ & \\
\hline & 3.2\%: "Turno distinto al que quería" & De las mujeres \\
\hline & 2.1\%: "Expulsado por indisciplina" & 23.8\%: "Se embarazó y tuvo un hijo" \\
\hline & 1.3\%: "La escuela quedaba muy lejos" & 22-9\%: "Se casó" \\
\hline
\end{tabular}

Fuente: ENDEMS, 2011, SEP-COPEMS, 2012.

Por su parte, en la Encuesta Nacional de Juventud (Instituto Mexicano de la Juventud, 2010), 42.6\% de los encuestados señalaron que el abandono escolar fue por causas escolares: a 15.9\% "la escuela le aburría", 13.1\%, "no quiso estudiar" y 9.6\%, "reprobó materias". 
La Subsecretaría de Educación Media Superior, para mejorar la permanencia, expandió las becas, ya que, de acuerdo con la ENDEMS, solo la octava parte de los desertores afirmaron que se beneficiaron con una; en cambio, casi la cuarta parte de los no desertores reportaron haberla recibido. Asimismo, ha continuado con varios componentes del programa Síguele del sexenio anterior, ahora convertido en el movimiento "Yo no abandono": seguimiento de los estudiantes en riesgo de abandonar la escuela (ahora en forma descentralizada en cada escuela), tutorías, diálogo con los padres y modificaciones al programa Construye-T, que fomenta las habilidades socioemocionales; también se brinda en internet una "Caja de herramientas" de doce manuales sobre temas relacionados con el fomento de la permanencia (Conaedu, 2015; Weiss, 2015a).

Asimismo, una de las medidas centrales fue comprometer a los directores de escuelas a elaborar un plan contra el abandono escolar en el que se responsabilizaran con determinadas metas a alcanzar. A la vez, se resaltaba la necesidad de flexibilizar normas y procesos administrativos para favorecer la permanencia escolar y la acreditación; en especial, asegurarse de que las normas y los reglamentos de control escolar no limitaran las oportunidades de los estudiantes para regularizarse, en particular no restringir, por cuestiones del ámbito administrativo, el número de evaluaciones no semestrales que pudiera tomar un alumno en cada periodo o a lo largo de su trayectoria académica, ya sea a través de cursos intersemestrales o exámenes extraordinarios o cualquier otra forma de regularización (Conaedu 2015; Weiss, 2015a).

En este rubro, efectivamente, hay cosas que mejorar; por ejemplo, en las escuelas que pertenecen a las modalidades de bachillerato tecnológico hay un "sistema" de control escolar que de manera automática reprueba a los estudiantes si no logran una asistencia de $80 \%$ y los da de baja del curso normal si reprueban más de dos asignaturas (Silva, 2016); en el Colegio de Bachilleres pueden reprobar máximo cuatro asignaturas (Saccone y Weiss, 2017) (en México el plan de estudios está organizado por semestres y la reprobación de determinado número de asignaturas no impide el avance al siguiente semestre.) Además, antes de ser reprobado en alguna asignatura, hay modalidades de recuperación diversas, como los exámenes "extraordinarios".

A pesar del esfuerzo, en el ciclo escolar 2015-2016 la tasa de abandono escolar para educación media superior representó 13.3\%, lejos de alcanzar la meta de 9\% del Programa Sectorial de Educación, y en contraste con la educación secundaria, que en el mismo ciclo escolar tenía una tasa de deserción de 4.2\% (Conaedu, 2017; INEE, 2017a). Esta cuestión se explica, en parte, por la incorporación de sectores poblacionales más pobres, más proclives a abandonar la escuela, así como por la lenta aceptación de la obligatoriedad.

En el trabajo que presentamos aquí nos centramos en los significados que tiene para los jóvenes asistir a la educación media superior y las razones por las que una parte no permanece. Antes, veamos algunas cuestiones metodológicas.

\section{NOTAS METODOLÓGICAS}

Nuestros datos estadísticos provienen de documentos oficiales, de los hallazgos empíricos de las tesis de maestría y doctorado, en su gran mayoría, que el autor 
dirige en la línea de investigación sobre Jóvenes y bachillerato, así como de estudios propios. Las referencias no buscan presentar un estado de conocimiento sobre el tema; se seleccionaron con el propósito de construir la línea argumentativa de este artículo. El escrito pone estos hallazgos en diálogo con los lineamientos de la política educativa, en especial, la obligatoriedad de la educación media superior y la flexibilización de la acreditación.

Nuestra línea de investigación cualitativa desde una perspectiva sociocultural sobre el tema de los jóvenes y el bachillerato comenzó con la tesis de maestría de Guerra y Guerrero acerca del significado del bachillerato para los jóvenes en dos instituciones: el Colegio de Ciencias y Humanidades (CCH) de la Universidad Autónoma de México y un Centro de Estudios Tecnológicos Industriales (CETI) en el Estado de México (Guerra, 2000; Guerra y Guerrero, 2004). Tapia (2015) abordó, en su tesis doctoral, el sentido de la escolaridad en comunidades rural-urbanas, en particular para estudiantes mujeres, en el Bajío del estado de Guanajuato.

En la investigación de Guerra y Guerrero (2004) había surgido el tema de la escuela como espacio juvenil, que fue profundizado en varias tesis. Ávalos (2007) observó en el CCH Sur la vida juvenil en el salón de clase y participó en las conversaciones de un grupo de jóvenes. Hernández (2007) profundizó en los significados del encuentro con amigos y novios y de la sexualidad. Grijalva (2011) investigó las identificaciones en grupos de pares a través de la ropa, la música y las actividades de estudio y diversión que desarrollan los jóvenes de un bachillerato público sostenido por la Universidad Autónoma de Sinaloa, en el puerto de Mazatlán. En su tesis doctoral, Guerrero (2008) indagó sobre la experiencia escolar en el CCH y mostró los peligros de la vida juvenil, pero también los puntos de retorno. Por su parte, Guerra (2008) estudió los recorridos escolares y laborales y sus virajes de jóvenes de sectores populares. Como apreciamos, las investigaciones se realizaron aprovechando la procedencia de los tesistas en diferentes tipos de escuelas de bachillerato en varias partes del país, aunque predominan instituciones de la Ciudad de México.

En estos estudios ya se problematizaban las seducciones de la vida juvenil. A partir de un encargo del Instituto Belisario Domínguez para emprender un estudio referente al abandono escolar (Weiss, 2015a), comenzamos a analizar los fenómenos de la falta de interés para el estudio y las insuficiencias académicas, así como los efectos de la obligatoriedad escolar en forma de la flexibilización de las exigencias y de los requisitos por parte de las instituciones. Estas temáticas fueron abordadas en la tesis de Saccone (2016) en el Colegio de Bachilleres y en la investigación de Silva (2016) en los bachilleratos tecnológicos agropecuarios que asesoramos. El encargo por parte del Instituto para la Evaluación de la Educación para analizar la nueva modalidad del telebachillerato comunitario (Weiss, 21017b) nos permitió enfocar la brecha entre las capacidades académicas de los estudiantes y las exigencias del plan y los programas de estudio.

Nuestra línea de investigación cualitativa se inscribe en buena parte en la tradición etnográfica del Cinestav, que propone "documentar o no documentado" (Rockwell 1987, p. 21), es decir, la vida escolar no documentada por los informes burocráticos, pero tampoco por las investigaciones pedagógicas, sociológicas, psicológicas o antropológicas que se acercan con un marco teórico preconcebido a partir del cual constatan la presencia o ausencia de determinados fenómenos. A la 
vez, las interpretaciones que realizamos son fundamentalmente hermenéuticas: se busca comprender las transcripciones de observaciones y entrevistas cualitativas como si fueran un texto de varias voces; comprender determinado significado en el contexto de otros significados simultáneos (como partes de un todo; el círculo hermenéutico como configuración de sentidos); y modificar nuestras anticipaciones de sentido hasta que logren ser interpretaciones pertinentes a lo que dice el texto (la espiral hermenéutica) (Weiss, 2017a).

En este texto, nos referimos de manera reiterada a significados y sentidos. La línea de diferenciación entre los dos conceptos es tenue. El término significados es más pertinente cuando hablamos de significados compartidos, como las representaciones sociales (Jodelet, 1984), y el de sentido, cuando destacamos más bien el sentido personal, como en la concepción de Vygotsky (González-Rey, 2010). El sentido personal del bachillerato se puede comprender a partir de la configuración de varios significados (Guerra y Guerrero, 2012), así como las razones o motivos del abandono escolar siempre son multidimensionales. En este artículo nos centramos en los significados y las razones compartidos.

\section{LOS SIGNIFICADOS DEL BACHILLERATO PARA LOS JÓVENES}

En las investigaciones analizadas, los significados más importantes para los estudiantes del CCH y del CETIS eran, respectivamente, el pase automático o el certificado para poder continuar sus estudios, aunque en el caso del bachillerato tecnológico no estaban tan seguros si iban a tener los medios económicos y los resultados académicos para continuar en el nivel superior (Guerra y Guerrero, 2004). Advertimos la expectativa de poder seguir estudiando una carrera a nivel superior aun en modalidades como el videobachillerato, por ejemplo, en una zona rural-urbana del estado de León; la mayoría se proponía, a la par del trabajo, estudiar en turnos vespertinos o sabatinos de alguna institución privada de paga (Tapia, 2015); incluso, en un bachillerato intercultural comunitario en un municipio de la sierra en el estado de Oaxaca un tercio de los estudiantes pensaban seguir estudiando (Weiss, 2006).

La posibilidad de encontrar un empleo formal era una motivación importante para los estudiantes del CETI. Una buena parte de ellos ya tenían experiencia laboral, pero en trabajos informales (Guerra, 2008). En el bachillerato intercultural comunitario, las mujeres pretendían un empleo en una tienda en una ciudad y los hombres, un trabajo en restaurantes en México y Estados Unidos; esto, en vez del trabajo tradicional como sirvientas en casa o como trabajadores agrícolas o ayudantes de albañil (Weiss, 2006).

Para muchos estudiantes de los sectores populares, el bachillerato significaba poder "ser alguien", es decir, tener prestigio social y alcanzar "una mejor vida", significados que eran también fomentados por los padres de familia. En los tres escenarios se encontraban también estudiantes que hablaban de apoyo de la familia, a veces de un hermano o hermana, y que esperaban poder retribuir en un futuro el esfuerzo de sus padres (Guerra, 2000; Tapia, 2015). Motivaciones como "haberlo logrado" y "demostrar que pude" estaban presentes en algunos.

Entre mujeres de sectores populares y con mayor fuerza en zonas rurales, destacaba la motivación de "superar la condición de género", como la llamaron Guerra y Guerrero (2004), por un lado, como reacción a la discriminación por parte de fa- 
miliares y vecinos y, por otro, en un sentido propositivo: ser en el futuro madres más instruidas. Tapia (2015) muestra que en el Bajío -en una zona de transición rural-urbana y de un catolicismo arraigado- aún se difunde entre padres y abuelas el "modelo cultural": "para qué estudias si de todos modos te vas a casar" (p. 247). Sin embargo, las madres apoyan a sus hijas para que asistan a la escuela para que se valgan por sí mismas en caso de que el marido no cumpla con las obligaciones. Estas jóvenes desplazan el noviazgo, el matrimonio y la procreación de hijos -usual en la edad de dieciséis a diecinueve años- hasta terminar cuando menos el bachillerato o una parte incluso planea "primero tengo mi carrera".

La sorpresa en el primer estudio sobre el significado del bachillerato, de Guerra y Guerrero (2004, 2012; también Guerra, 2000), fueron respuestas como "voy a la escuela para encontrarme con mis amigos", "ahí veo a mi novio" y "en casa me aburro". Clasificamos este tipo de expresiones con la categoría "la escuela como espacio juvenil", segunda en importancia por encima de los beneficios de "la formación académica" en el caso de los estudiantes del CCH y la tercera después de "un mejor empleo" en los estudiantes del CETI.

El tema de la escuela como espacio juvenil ha sido profundizado en varias tesis (Weiss et al., 2009). Ávalos (2007) observó en el CCH Sur la vida juvenil en el salón de clase y participó en las conversaciones de un grupo de jóvenes. La vida juvenil no se limita a los tiempos antes, entre y después de clases, sino que se despliega también durante la clase y sustrae tiempo a la actividad escolar para bromas o intercambios (de tonos y juegos en celulares, música y cómics, entre otros) hasta las diversas conversaciones que surgen espontáneamente sobre los temas más variados, en especial durante los trabajos grupales. Las diversas posibilidades de vida juvenil suelen competir con los requerimientos escolares. Grijalva (2011) encuentra, en el patio de un bachillerato dependiente de la universidad en el puerto de Mazatlán, grupos de alumnos que privilegian el trabajo escolar (los menos), los que privilegian la diversión y la vida juvenil (los más) y los que combinan de modo estratégico la diversión con el trabajo escolar.

En varios estudios (Hernández, 2007; Ávalos, 2007; Grijalva, 2011) observamos la relevancia de la sociabilidad (Simmel 2002), del disfrute de estar juntos con compañeros, amigos y novios. También, lo que Maffesoli (2004) llama la "socialidad", el gusto por las fiestas y el exceso, que está presente en el relajo, los juegos físicos y las bromas; las fiestas y las actividades musicales son de lo más importante para muchos jóvenes; en la escuela se comentan las experiencias en los últimos actos y fiestas; además, se planea la asistencia a los próximos. Sin embargo, las experiencias de los jóvenes no se limitan al hedonismo ni a la sociabilidad; la convivencia juvenil y los encuentros con compañeros, amigos y parejas significan más que solo "vibrar juntos".

Diferentes grupos de estudiantes de bachillerato desarrollan sus propias normas y valores respecto al comportamiento con los maestros, a la dedicación a las tareas, al look, a la música aceptable y al tipo de diversiones preferidas (Grijalva, 2011; Ávalos, 2007). En este sentido, estamos de acuerdo con Coleman (1961) de una socialización intrageneracional (en oposición a la intergeneracional pretendida de los adultos). Desde una perspectiva crítica, constatamos que en cuestiones de look los estudiantes se identifican con determinadas culturas juveniles y sus dictados de moda; en términos de Foucault, se constituyen como sujetos en tanto son sujetados por los discursos de las industrias de consumo juveniles, pero también es interesante observar el desarrollo de un 
gusto propio entre las mujeres estudiantes de más edad en el bachillerato en el puerto de Mazatlán: ellas destacan la forma personal en la que combinan ciertos atuendos y accesorios al considerar en sus decisiones las características de su cuerpo y personalidad (Grijalva, 2011).

Concebimos el proceso de subjetivación como de interiorización y apropiación de normas y valores y del desarrollo de la capacidad de reflexionar desde el self sobre las distintas demandas y la posición propia en ellas; de ahí nacen la emancipación de las normas y los valores dominantes y el desarrollo de gustos, intereses, capacidades y normas propias que fundamentan la agencia del sujeto, la capacidad humana de hacer y decidir (Weiss, 2015b). Este proceso se desarrolla a través de diversas prácticas en interacción con otros en la edad juvenil, sobre todo con compañeros, amigos y novios, y mediante la conversación sobre las vivencias.

A diferencia de los estudios sobre culturas juveniles (Reguillo, 2000), en los cuales destaca el "otro antagonista", en los nuestros prevalece el "otro semejante". El "otro" más importante es la pareja amorosa o sexual. Sin duda, el tiempo del bachillerato es, para la gran mayoría de jóvenes, un tiempo de vivir relaciones eróticas y sexuales. Los estudiantes en el CCH en la Ciudad de México establecen diferentes tipos de relaciones. El conocimiento práctico del "otro" pasa por la sensualidad corporal, y también por compartir, abrirte al otro, por el apoyo recíproco, el ensayo y el error. En las relaciones amorosas, los jóvenes aprenden a manejar los vínculos sociales de intimidad, confianza, cuidado del otro e incertidumbre amorosa, así como las capacidades de expresar los afectos, descubrir otras maneras de sentir y atender las necesidades del otro, como muestra Hernández (2007). Las relaciones eróticas son más reservadas, pero observables en el Bachillerato Intercultural Comunitario, que constituye un espacio público inédito para el encuentro entre jóvenes mujeres y hombres en los municipios indígenas (Hernández y Weiss, 2010).

El tiempo que sustraen los jóvenes a lo académico para estar con sus compañeros, amigos y novios no se puede considerar solo como una pérdida; constituye un tiempo/espacio valioso para la socialización entre pares y el proceso de subjetivación, es decir, la formación de la persona.

La condición de ser joven y la de ser estudiante no necesariamente se oponen. Así lo muestran los estudiantes "estrategas" -sobre la importancia de la estrategia entre estudiantes de bachillerato en Francia, véase Dubet y Martuccelli (1998)- que disfrutan de su vida con sus pares a la vez que invierten el tiempo y esfuerzo necesarios para aprobar materias u obtener buenas calificaciones para poder tener acceso a las carreras universitarias de su elección. Sin embargo, la dedicación a la vida juvenil por encima de los estudios implica también peligros como veremos en el próximo apartado sobre el abandono escolar y, para algunos estudiantes, la convivencia juvenil y la obligación de asistir a la escuela se convierten en los significados dominantes.

\section{EL ABANDONO ESCOLAR DESDE LA PERSPECTIVA DE ESTUDIOS CUALITATIVOS}

Los significados positivos atribuidos al bachillerato no aparecen con igual fuerza en todos los alumnos. Hay estudiantes que solo van a la escuela para encontrarse con sus compañeros, amigos o parejas, o asisten porque sus familias los envían. 
Guerra (2008), al estudiar los recorridos escolares y laborales de los estudiantes del bachillerato tecnológico, distingue entre quienes se adhirieron a la cultura escolar y quienes nunca desarrollaron un apego a los valores y las normas de la escuela y consideraron más importante el trabajo o la vida en pareja o los hijos, en el caso de las mujeres, y los que entraron en conflicto con los valores y las normas de la escuela y prefirieron la vida juvenil en la calle. En las situaciones de abandono escolar, las razones o los motivos se entrelazan: el joven tiene dificultades académicas; en su barrio o en la escuela se une a un grupo que se dedica poco o nada a los estudios $y$, en consecuencia, falta a clases, no atiende sus deberes y reprueba crecientemente materias; en este contexto, cualquier incidente, una riña, una enfermedad, una oferta de trabajo temporal atractiva, otra reprobación o una mala palabra de un maestro impulsan al joven a abandonar la escuela o a ser expulsado.

Algunos de ellos cambian su curso de vida, reflexionan y se dedican otra vez a estudiar o regresan a la escuela. A partir de malas experiencias, se hacen cargo de sí mismos y de su futuro, se "vuelven responsables" y "maduran" como ellos mismos dicen (Guerra 2012). Sin embargo, no se les readmite fácilmente en el mismo sistema ni se le reconocen los semestres o materias aprobados, aun en la actualidad, a pesar de haber sido la transportabilidad de las acreditaciones uno de los objetivos principales de la Reforma Integral de la Educación Media Superior y del establecimiento de un marco curricular común. En forma reciente se abrió la opción del bachillerato en línea y se expandió la "prepa abierta", que resultan factibles para jóvenes que ya han adquirido ciertas bases para el autoestudio.

Los recorridos escolares suelen ser "trayectorias" progresivas y lineales más bien entre los estudiantes de clase media y alta. En cambio, entre los de sectores populares urbanos se encuentran con más frecuencia los recorridos fragmentados, caracterizados por la reprobación, el abandono y, en algunos casos, por los intentos reiterados de obtener el certificado de bachillerato (Guerra, 2012). Muchos jóvenes de clases populares tienen que trabajar para contribuir con sus ingresos a la supervivencia de la familia; otros prefieren trabajar para poder darse el gusto de comprarse unos tenis o un smartphone. Muchas mujeres tienen que quedarse en casa para cuidar hermanos menores o enfermos y contribuir a las tareas productivas o reproductivas del hogar. Asimismo -aunque estos patrones están cambiando muy rápidamente-, en la cultura popular ir a trabajar después de la secundaria todavía es considerada una vía legítima para hacerse hombre, y quedarse en la casa para ayudar a la familia, una forma protegida de hacerse mujer. Por otro lado, se presentan las ya mencionadas seducciones de la vida juvenil que dificultan la permanencia en la escuela.

En la actualidad, casi todos los alumnos que terminan la secundaria comienzan a estudiar el nivel medio superior. Sin embargo, hay que tomar con cuidado la cifra oficial de absorción, que fue de 99.5\% en 2011-2012, ya que las estadísticas escolares suelen sobrevalorar la absorción al contar en el numerador dos veces a los que se inscriben en determinado ciclo escolar en dos instituciones diferentes, primero en una y luego en otra (Weiss, 2015a). Los hijos de las familias más pobres no terminan la educación básica y no entran al bachillerato, como lo revela un estudio sobre el telebachillerato en una aldea campesina en las Cañadas de Oaxaca (Auli, en proceso). 
En el estudio cualitativo de Silva (2016) sobre el abandono escolar en cuatro bachilleratos tecnológicos agropecuarios en los estados de Morelos y Hidalgo, dos con bajo abandono y dos con alto abandono, la falta de recursos fue señalada por menos de la mitad de los entrevistados como una de las razones del abandono. Ahí observamos el impacto positivo de la política de becas; en las cuatro escuelas visitadas, entre 80 y $90 \%$ de los estudiantes tenían becas. Hoy en día y con apoyo de las becas, la gran mayoría de familias están dispuestas a afrontar el gasto de enviar a sus hijos a la escuela; los problemas surgen cuando salen imprevistos.

Al mismo tiempo, casi todos los jóvenes entrevistados que abandonaron la escuela señalaron que habían acumulado reprobaciones y el resto decían que no tenían interés en los estudios. Las causas principales para la reprobación eran: "las inasistencias a la escuela" o "por no entrar a clase": "me la pasaba en las canchas"; también reprobaban por "no entregar tareas" (Silva, 2016, p. 65). Unos señalaban, además, que "no entendía y no ponía atención", que el profesor "iba muy rápido" o que reprobaron exámenes; a veces también por mal comportamiento dentro del aula: "el profe me sacó de la clase" (Silva, 2016, p. 66). Silva concluye que la razón principal para el absentismo y no entregar trabajos y tareas es la falta de interés en el estudio: "le dije a mi novia que no quería estudiar, y se lo dijo a mi mamá, pero mi mamá me manda a la escuela" (Silva, 2016, p. 71). Debemos agregar a los significados del bachillerato para los jóvenes uno más: la obligatoriedad.

\section{LA FLEXIBILIZACIÓN DE LA REPROBACIÓN}

La Subsecretaría de Educación Media Superior ha vinculado el otorgamiento de recursos adicionales a los planteles -incluso los recursos no relacionados con el abandono escolar- al logro de bajar las tasas de abandono. En este contexto, las escuelas han comenzado a modificar sus reglamentos de regularización. En el bachillerato agropecuario, muchas escuelas han dejado de reportar todas las ausencias de los alumnos al "sistema" para circunvenir la reprobación automática. "Ahora los maestros ya no le dan tanto peso a la asistencia, porque la reforma dice que la asistencia ya no es tan de peso", asegura un director (Silva, 2016, p. 64). En el Colegio de Bachilleres existe desde el sexenio anterior el Programa de Acreditación Intensivo para los estudiantes que reprobaron por acumulación de ausencias, el cual puede cursarse en modalidad intersemestral o sabatino. Saccone (2016) encontró estudiantes que prefieren esta modalidad porque requiere poco tiempo. Las más de las veces no es porque trabajen; en su gran mayoría, los jóvenes -aun los del turno vespertinono trabajan o lo hacen en "chambas" y trabajos de medio tiempo -de "empacadora" o "cerillo" en un supermercado o en fines de semana en un restaurante (Saccone, 2016; Saccone y Weiss, 2017).

Las escuelas, a su vez, condicionan a los profesores para que flexibilicen sus criterios de calificación. En un subsistema federal de bachillerato general los profesores solo pueden beneficiarse del Programa de Estímulos al Desempeño del Personal Docente si son comparables con los promedios del plantel en la asignatura correspondiente los porcentajes de permanencia y de aprobación de sus estudiantes y el número de calificaciones finales entre 8 y 10 (Colegio de Bachilleres, 2015, p. 4). En las escuelas agropecuarias de uno de los estados visitados por Silva (2016), los 
coordinadores decidieron que ningún profesor debe reprobar más de 10\% de los estudiantes inscritos en su clase. Hay discusiones y tensión entre los profesores. Algunos, sobre todo los más antiguos, no están dispuestos a cambiar sus formas de trabajar ni sus exigencias.

Una de las razones principales del abandono escolar es la acumulación de materias reprobadas y, como vimos, las ausencias y la falta de entregar trabajos son causas principales para la reprobación. Hay estudiantes que asisten a la escuela porque sus familias los envían o para encontrarse con amigos, pero no entran a clase. En el Colegio de Bachilleres podían salir y regresar a la escuela durante la jornada escolar; ahora solo pueden salir de las instalaciones al final de la jornada, pero sin que haya quien los obligue a entrar a clase. La disposición de tener que quedarse dentro de las instalaciones es resentida como una coerción de su libertad como jóvenes por unos, aunque también hay quienes reconocen que esas medidas les ayudan (Saccone, 2016).

Los estudiantes del Colegio de Bachilleres relatan en entrevistas que los docentes, en general, solicitan pocas tareas y que a algunos les basta el hecho de que se entreguen -muchas veces copiadas de compañeros o de páginas de internet- y que no las revisan. Los jóvenes saben que con algunos docentes más que otros pueden negociar el criterio de acreditación vinculado a la entrega en determinado tiempo y forma de tareas y trabajos escolares, y que en estas negociaciones suelen ganar. "Se percibe cierta tendencia a la flexibilidad y permisividad, sobre todo en lo referido a la asistencia y la puntualidad en el ingreso a clases, pero también en la casi nula exigencia de tareas para realizar en horario extraescolar y la evaluación fundamentalmente a partir de los trabajos realizados en clase, reduciendo la toma de exámenes" (Saccone, 2016, p. 76). Este tipo de fenómenos no se observa solo en México; en Argentina un estudio de Meo (2011), que logró mucha atención, analizó la estrategia de "zafar" (hacer un esfuerzo mínimo para aprobar) como disposición general de los estudiantes del secundario.

\section{LAS DEFICIENCIAS EN LA FORMACIÓN ACADÉMICA}

El abandono escolar se relaciona fuertemente con la reprobación y esta, con las deficiencias en la formación académica de los alumnos. En el estudio de Silva (2016) sobre el abandono escolar en bachilleratos en los estados de Hidalgo y Morelos, algunos jóvenes mencionan de manera explícita que "no entendían a los profesores". También podemos pensar que muchos de los jóvenes en riesgo de abandono que afirman que "no tienen interés en la escuela" o "no tienen ganas de entrar a clases" y pasan el día en las canchas u otra parte de las instalaciones, tienen problemas para comprenderlas. Estos jóvenes suelen provenir de familias que alcanzaron niveles mínimos de escolaridad y en su trayectoria escolar muchos estudiaron en escuelas deficientes, que ahondan más que compensar las deficiencias culturales y académicas.

Diversas mediciones del aprendizaje, internacionales como el Programa para la Evaluación Internacional de Alumnos (PISA) y nacionales como el Plan Nacional de Evaluación de Aprendizajes (Planea), muestran que los alumnos de bachillerato no logran los aprendizajes de este nivel escolar. Los resultados del Planea (INEE, 2017b) revelan que la mitad de los estudiantes del último año de la educación media superior solo logran el nivel más bajo de la medición (nivel I) y quedan incluso por debajo del nivel de los estándares de la educación básica. 
con énfasis en las matemáticas, las ciencias, la historia y el lenguaje. En la educación tecnológica, que atiende el tercio restante, han aumentado las materias académicopropedéuticas y disminuido el número de materias tecnológicas (Weiss, 2015a).

El telebachillerato comunitario, que es un nuevo subsistema creado en el actual sexenio para incorporar estudiantes en comunidades rurales pequeñas, se rige por el plan de estudios del bachillerato general. En el "Mapa curricular del Bachillerato General para telebachillerato comunitario con un enfoque educativo basado en el desarrollo de competencias" (ver tabla 3), estas últimas solo aparecen en el título. La especificidad del telebachillerato comunitario queda confinada al espacio de una asignatura de las ocho por semestre que se imparte del tercero al sexto semestre: Desarrollo comunitario. Por cierto, este espacio está previsto para la formación del trabajo en otras modalidades del bachillerato general. En cambio, sigue firme el bloque de materias propedéuticas para el estudio de la educación superior durante el quinto y sexto semestre: Probabilidad y estadística, Derecho, Ciencias de la comunicación y Ciencias de la salud (Weiss, 2017b).

Tabla 3. Mapa curricular del Bachillerato General para telebachillerato comunitario con un enfoque educativo basado en el desarrollo de competencias

\begin{tabular}{|c|c|c|c|c|c|c|c|c|c|c|c|c|c|c|c|c|c|}
\hline \multicolumn{3}{|c|}{ PRIMER SEMESTRE } & \multicolumn{3}{|c|}{ SEGUNDO SEMESTRE } & \multicolumn{3}{|c|}{ TERCER SEMESTRE } & \multicolumn{3}{|c|}{ CUARTO SEMESTRE } & \multicolumn{3}{|c|}{ QUINTO SEMESTRE } & \multicolumn{3}{|c|}{ SEXTO SEMESTRE } \\
\hline ASIGNATURA & $\mathrm{H}$ & $\mathrm{C}$ & ASIGNATURA & $\mathrm{H}$ & $\mathrm{C}$ & ASIGNATURA & $\mathrm{H}$ & $\mathrm{C}$ & ASIGNATURA & $\mathrm{H}$ & $\mathrm{C}$ & ASIGNATURA & $\mathrm{H}$ & $\mathrm{C}$ & ASIGNATURA & $\mathrm{H}$ & $\mathrm{C}$ \\
\hline Matemáticas I & 5 & 10 & Matemáticas II & 5 & 10 & $\begin{array}{c}\text { Matemáticas } \\
\text { III }\end{array}$ & 5 & 10 & $\begin{array}{l}\text { Matemáti- } \\
\text { cas IV }\end{array}$ & 5 & 10 & & & & Filosofía & 4 & 8 \\
\hline Química I & 5 & 10 & Química II & 5 & 10 & Biología I & 4 & 8 & Biología II & 4 & 8 & Geografía & 3 & 6 & $\begin{array}{l}\text { Ecología } \\
\text { y medio } \\
\text { ambiente }\end{array}$ & 3 & 6 \\
\hline $\begin{array}{c}\text { Ética y valo- } \\
\text { res I }\end{array}$ & 3 & 6 & $\begin{array}{c}\text { Ética y valores } \\
\text { II }\end{array}$ & 3 & 6 & Física I & 5 & 10 & Física II & 5 & 10 & $\begin{array}{c}\text { Historia } \\
\text { universal } \\
\text { contemporánea }\end{array}$ & 3 & 6 & $\begin{array}{c}\text { Metodología } \\
\text { de la } \\
\text { investigación }\end{array}$ & 3 & 6 \\
\hline $\begin{array}{l}\text { Introducción } \\
\text { a las ciencias } \\
\text { sociales }\end{array}$ & 3 & 6 & $\begin{array}{l}\text { Historia de } \\
\text { México I }\end{array}$ & 3 & 6 & $\begin{array}{l}\text { Historia de } \\
\text { México II }\end{array}$ & 3 & 6 & $\begin{array}{l}\text { Estructura } \\
\text { socioeco- } \\
\text { nómica de } \\
\text { México }\end{array}$ & 3 & 6 & *Derecho I & 3 & 6 & *Derecho II & 3 & 6 \\
\hline $\begin{array}{c}\text { Taller de } \\
\text { lectura y } \\
\text { redacción I }\end{array}$ & 4 & 8 & $\begin{array}{c}\text { Taller de } \\
\text { lectura y } \\
\text { redacción II }\end{array}$ & 4 & 8 & Literatura I & 3 & 6 & Literatura II & 3 & 6 & $\begin{array}{c}\text { *Ciencias de la } \\
\text { comunicación } \\
\text { I }\end{array}$ & 3 & 6 & $\begin{array}{c}{ }^{*} \text { Ciencias de la } \\
\text { comunicación } \\
\text { II }\end{array}$ & 3 & 6 \\
\hline \multirow[t]{2}{*}{$\begin{array}{c}\text { Lengua } \\
\text { adicional al } \\
\text { español I }\end{array}$} & 3 & 6 & $\begin{array}{c}\text { Lengua } \\
\text { adicional al } \\
\text { español II }\end{array}$ & 3 & 6 & $\begin{array}{c}\text { Lengua } \\
\text { adicional al } \\
\text { español III }\end{array}$ & 3 & 6 & $\begin{array}{c}\text { Lengua } \\
\text { adicional al } \\
\text { español IV }\end{array}$ & 3 & 6 & $\begin{array}{c}\text { *Ciencias de la } \\
\text { salud I }\end{array}$ & 3 & 6 & $\begin{array}{c}\text { Ciencias de la } \\
\text { salud II }\end{array}$ & 3 & 6 \\
\hline & & & & & & \multirow[t]{2}{*}{$\begin{array}{l}{ }^{* *} \text { Desarrollo } \\
\text { comunitario I }\end{array}$} & \multirow[t]{2}{*}{7} & \multirow[t]{2}{*}{14} & \multirow{2}{*}{$\begin{array}{c}\text { **Desarrollo } \\
\text { comunita- } \\
\text { rio II }\end{array}$} & \multirow[t]{2}{*}{7} & \multirow[t]{2}{*}{14} & $\begin{array}{l}\text { *Probabilidad } \\
\text { y estadística }\end{array}$ & 3 & 6 & $\begin{array}{l}\text { *Probabilidad } \\
\text { y estadística }\end{array}$ & 3 & 6 \\
\hline \multirow[t]{4}{*}{$\begin{array}{c}\text { Actividades } \\
\text { paraescolares }\end{array}$} & 4 & & $\begin{array}{c}\text { Actividades } \\
\text { paraescolares }\end{array}$ & 4 & & & & & & & & \multirow{2}{*}{$\begin{array}{c}* * \text { Desarrollo } \\
\text { comunitario } \\
\text { III }\end{array}$} & \multirow[t]{2}{*}{7} & \multirow[t]{2}{*}{14} & \multirow{2}{*}{$\begin{array}{c}\text { ** Desarrollo } \\
\text { comunita- } \\
\text { rio IV }\end{array}$} & \multirow[t]{2}{*}{7} & \multirow[t]{2}{*}{14} \\
\hline & 27 & 46 & & 27 & 46 & $\begin{array}{c}\text { Actividades } \\
\text { paraescolares }\end{array}$ & 3 & & $\begin{array}{c}\text { Actividades } \\
\text { paraesco- } \\
\text { lares }\end{array}$ & 2 & & & & & & & \\
\hline & & & & & & & 33 & 60 & & 32 & 60 & $\begin{array}{c}\text { Actividades } \\
\text { paraescolares }\end{array}$ & 4 & & $\begin{array}{c}\text { Actividades } \\
\text { paraescolares }\end{array}$ & 3 & \\
\hline & & & & & & & & & & & & & 29 & 50 & & 32 & 58 \\
\hline
\end{tabular}

*Componente de formación propedéutica

*Componente de formación para el trabajo por módulos bajo enfoque de competencia laboral

Fuente: SEP-DGB. http://www.dgb.sep.gob.mx/servicios-educativos/telebachillerato/normatividad/Mapa_CURRICULAR_tbc_2014.pdf

Weiss. Los significados del bachillerato para los jóvenes y la permanencia escolar en México Sinéctica 51 www.sinectica.iteso.mx 
En este plan hay cuatro cursos seriados de Matemáticas. En el programa de Matemáticas IV se aborda cálculo diferencial. Las autoridades educativas olvidan los resultados de las mediciones de aprendizaje al prescribir cálculo diferencial a estudiantes que, como vimos en la tabla 2, en 66\% "tienen dificultades para realizar operaciones con fracciones y operaciones que combinen incógnitas o variables (representadas con letras), así como para establecer y analizar relaciones entre dos variables". Al docente de esta asignatura en las escuelas agropecuarias en uno de los estados visitados por Silva (2016) le indican que solo debe reprobar 10\% de sus estudiantes.

\section{LAS POLÍTICAS EDUCATIVAS QUE REQUERIMOS (A MODO DE CONCLUSIÓN)}

Nuestra línea de investigación comenzó cuando el bachillerato aún no era obligatorio; en ese entonces -y al inicio del artículo- señalamos los sentidos positivos que otorgan los estudiantes al bachillerato. Destacamos su importancia como lugar de encuentro juvenil que permite conocer a otros, en especial parejas. Asimismo, hicimos hincapié en que el tiempo que dedican a la convivencia y las conversaciones no debe considerarse solo como tiempo perdido, sino como tiempo para adquirir experiencias y para el crecimiento. Sin embargo, nuestras investigaciones posteriores, centradas en el tema del abandono escolar, dirigieron nuestra mirada a los problemas de falta de interés en los estudios y a las deficiencias académicas de una parte de los estudiantes, así como a la creciente flexibilización de las exigencias y los criterios de acreditación y calificación por parte de las instituciones educativas en el contexto de la obligatoriedad escolar.

Las políticas actuales buscan presentar un país moderno a los potenciales inversores internacionales, con estadísticas que muestran una fuerza de trabajo escolarizada a nivel de bachillerato y con planes de estudio de altas exigencias académicas. La exigencia a las escuelas y docentes de disminuir el abandono escolar se traduce no solo en la flexibilización de los requisitos de acreditación, sino en el relajamiento de las exigencias académicas y escolares. La mayoría de los estudiantes del nivel medio (secundaria y bachillerato) siempre han sido estrategas de su tiempo: según las exigencias escolares, invierten tiempo y esfuerzo a las tareas escolares; el tiempo restante lo dedican a sus intereses juveniles (Dubet y Martuccelli, 1998).

La política de obligatoriedad de la educación media superior, sin cambios en la estructura curricular, fomenta la simulación del aprendizaje y la disminución de la calidad educativa. Este fenómeno ha estado ocurriendo en las dos últimas décadas en la educación secundaria y actualmente está pasando en la educación media superior. En términos generales, es evidente que hay diferencias de calidad entre las modalidades educativas, entre los planteles y entre los grupos escolares matutinos y vespertinos que reproducen las diferencias socioculturales (Blanco, Solís y Robles, 2014; Ramírez y Remedi, 2015) y entre los estudiantes hay diferencias en las aspiraciones y los esfuerzos.

Frente a este panorama, cabe preguntarnos ¿les preocupa a los políticos y a las autoridades educativas la baja calidad de los aprendizajes? Tal vez algo, ahí está el padrón de calidad del Sistema Nacional de Bachillerato (Conaedu, 2017). No obstante, en un país donde hay pocos empleos formales de calidad a ofrecer a los egresados y donde acecha el peligro de que los jóvenes caigan en las redes del narcotráfico, 
es preferible que estén resguardados en las escuelas y no expuestos en la calle. La "escuela de la ignorancia" como guardería, como lo plantea Michéa (2002), pero hay que resaltar también los aspectos positivos: constituyen un lugar de encuentro seguro para la construcción de relaciones de pareja.

Por otra parte, la expansión del nivel se ha realizado con presupuestos restringidos (Sergio, 2015; INEE, 2017a). Lograr la obligatoriedad del nivel medio superior con calidad exigiría aumentos presupuestarios sustanciales, mejores salarios y empleos de tiempo completo para los docentes, los cuales, en su mayoría, trabajan por tiempos parciales en diferentes secundarias y bachilleratos para completar una jornada excesiva que no les permite preparar las clases y revisar con cuidado los trabajos de los alumnos.

También se necesitan recursos para una inducción a la docencia durante dos años como mínimo, con tutores calificados, para una formación docente continua, y no programas esporádicos como el diplomado en Formación Docente para la Educación Media Superior, que se ofreció en el sexenio pasado, pero solo transmitió el nuevo discurso educativo: el enfoque de competencias, pero no como se traduce en la enseñanza en el salón de clases, o los cursos en línea que se brindan en el sexenio actual.

Se requiere una verdadera reforma curricular en la mayoría de los planes de estudio para tender puentes entre la propuesta escolar y la vida juvenil. Acercar la escuela a la cultura juvenil no quiere decir renunciar a la transmisión cultural de la herencia de generaciones anteriores ni a la formación de las siguientes generaciones en los conocimientos científicos y culturales que las sociedades consideran útiles. Más bien habrá que buscar que la escuela se convierta en mediador entre las culturas juveniles y las tradiciones culturales, en una instancia que permita el diálogo entre pasado, presente y futuro, que constituya un puente intergeneracional. Un plan de estudios que, en vez de reproducir las asignaturas del siglo XIX, fomente de manera efectiva las competencias de comunicación y socioculturales.

Habrá que desarrollar formas eficaces para convocar y apasionar a los jóvenes en el conocimiento académico; proponer a los estudiantes situaciones menos "escolares" y más significativas en términos de vida, así como situaciones auténticas que desarrollen procesos de aprendizaje. Para lograrlo, es importante un desarrollo más vigoroso de las didácticas específicas de las disciplinas y la producción de materiales pertinentes. Por otro lado, se pueden incorporar múltiples actividades juveniles que se apoyen en el uso de diversas tecnologías, tradicionales o nuevas, como parte de una concepción novedosa de educación tecnológica, artística y física para todos. Pensamos, por ejemplo, en pequeños proyectos productivos o comunitarios, en la edición de videos, la animación y la edición musical, y en grupos de capoeira o yoga. 


\section{REFERENCIAS BIBLIOGRÁFICAS}

Auli, Iván (en proceso). Escolaridad y trabajo de jóvenes rurales. Un estudio etnográfico en San Juan Coyula, Oaxaca. Tesis de maestría. DIE-Cinvestav, México.

Ávalos, Job. (2007). La vida juvenil en el bachillerato. Una mirada etnográfica. Tesis de maestría. DIE-Cinvestav, México.

Blanco, Emilio, Solís, Patricio y Robles, Héctor (2014). Caminos desiguales. Trayectorias educativas y laborales de los jóvenes en la Ciudad de México. México: INEE/El Colegio de México.

Colegio de Bachilleres (2015). Convocatoria. Programa de Estímulos al Desempeño del Personal Docente 2015-2016. México. Recuperado de http://www. cbachilleres.edu.mx/cb/convocatorias/2015/Personal_Docente/convocatoria_estimulos_2015-2016.pdf

Coleman, James (1961). The adolescent society. Nueva York, EUA: Free Press.

Conaedu. Capítulo Media Superior (2017). LVII reunión-Capítulo EMS. Recuperado de http://www.sems.gob.mx/en_mx/sems/lvii-reunion-consejo-nacional-autoridades-educativas-media-superior

Conaedu. Capítulo Media Superior (2015). XLII reunión-Capítulo EMS. Recuperado de http://www.sems.gob.mx/work/models/sems/Resource/12125/52_ CONAEDU_EMS.pdf

Diario Oficial de la Federación (2012). Decreto por el que se declara reformado el párrafo primero; el inciso c) de la fracción II y la fracción V del artículo 3o., y la fracción I del artículo 31 de la Constitución Política de los Estados Unidos Mexicanos. 9 de febrero. Recuperado de http://www.dof.gob.mx/ nota_detalle.php?codigo $=5233070 \&$ fecha $=09 / 02 / 2012 \&$ print $=$ true

Dubet, Francois y Martuccelli, Danilo (1998). En la escuela. Sociología de la experiencia escolar. Buenos Aires, Argentina: Losada.

González-Rey, F. L. (2010). Las categorías de sentido, sentido personal y sentido subjetivo en una perspectiva histórico-cultural: un camino hacia una nueva definición de subjetividad. Universitas Psychologica, vol. 9, núm. 1, pp. 241-253.

Grijalva, Olga (2011, enero-junio). La diversión y el trabajo académico como fuentes de las identificaciones de los jóvenes en sus grupos de pares. $C P U$ e, Revista de Investigación Educativa 12. Recuperado de https://www.uv.mx/ cpue/num12/inves/grijalva-diversion-trabajo.html

Guerra, María Irene (2012). Recorridos escolares. Jóvenes de sectores populares y escuela: encuentros y desencuentros a lo largo de la vida. En Eduardo Weiss (coord.). Jóvenes y bachillerato (pp. 243-266). México: Asociación Nacional de Universidades e Instituciones de Educación Superior.

Guerra, María Irene (2008). Trayectorias escolares y laborales de jóvenes de sectores populares: un abordaje biográfico. Tesis de doctorado. DIE-Cinvestav, México. Publicado como libro en 2009. Trayectorias formativas y laborales de jóvenes de sectores populares. Un abordaje biográfico. México: Asociación Nacional de Universidades e Instituciones de Educación Superior.

Guerra, María Irene (2000). ¿Qué significa estudiar el bachillerato? La perspectiva de los jóvenes en diferentes contextos socioculturales. Revista Mexicana de Investigación Educativa, vol. 5, núm. 10, pp. 243-272. 
Guerra, María Irene y Guerrero, María Elsa (2012). ¿Para qué ir a la escuela? Los significados que los jóvenes atribuyen a los estudios de bachillerato. Capítulo I. En Eduardo Weiss (coord.). Jóvenes y bachillerato (pp. 33-62). México: Asociación Nacional de Universidades e Instituciones de Educación Superior.

Guerra, María Irene y Guerrero, María Elsa (2004). Qué sentido tiene el bachillerato. Una visión desde los jóvenes. México: Universidad Pedagógica Nacional.

Guerrero, María Elsa (2008). Los estudios de bachillerato: un acercamiento a la perspectiva juvenil. Tesis de doctorado. DIE-Cinvestav, México.

Hernández, Joaquín (2007). La formación de la identidad en el bachillerato: reflexividad y marcos morales. Tesis de doctorado. DIE-Cinvestav, México. Publicado como libro en 2008. El trabajo de los estudiantes de bachillerato: reflexividad, voces y marcos morales. México: Universidad Pedagógica Nacional.

Hernández, Joaquín y Weiss, Eduardo (2010, octubre). Amor y sexualidad entre jóvenes de la escuela media en México. Cuadernos de Educación, año VIII, núm. 8, pp. 245-256.

Instituto Mexicano de la Juventud (2010). Encuesta Nacional de la Juventud. Recuperado de http://sic.conaculta.gob.mx/centrodoc_documentos/292.pdf

INEE (2017a, noviembre). Directrices para mejorar la permanencia escolar en la educación media superior. Recuperado de http://publicaciones.inee.edu.mx/ buscadorPub/P1/F/105/P1F105.pdf

INEE (2017b). Plan Nacional de Evaluación de Aprendizajes (Planea). Resultados nacionales. Educación media superior. Lenguaje y comunicación, matemáticas. Textos de divulgación. Recuperado de http://publicaciones.inee.edu.mx/ buscadorPub/P2/A/328/P2A328.pdf

INEE (2017c). Plan Nacional de Evaluación de Aprendizajes (Planea). 3ํo de secundaria. Lenguaje y comunicación, matemáticas. Textos de divulgación. Recuperado de http://publicaciones.inee.edu.mx/buscadorPub/ P2/A/336/P2A336.pdf

INEE (2016). Panorama educativo de México 2015. Recuperado de http://publicaciones.inee.edu.mx/buscadorPub/P1/B/114/P1B114.pdf

Jodelet, Denise (1984). Les représentations sociales: phénomènes, concept et théorie. En S. Moscovici (ed.). La psychologie sociale (pp. 357-378). París: PUF.

Maffesoli, Michel (2004). El tiempo de las tribus. El ocaso del individualismo en las sociedades posmodernas: México: Siglo XXI.

Meo, Analía Inés (2011). Zafar, so good. Middle-class students, school habitus and secondary schooling in the city of Buenos Aires (Argentina). British Journal of Sociology of Education, vol. 32, núm. 3, pp. 349-367. Recuperado de http://dx.doi.org/10.1080/01425692.2011.559338

Michéa, Jean Claude (2002). La escuela de la ignorancia y sus condiciones modernas. Madrid, España: Ediciones Acuarela.

Ramírez, Rodolfo y Remedi, Eduardo (2015). La calidad de la educación media superior: ¿qué sabemos hoy? En Rodolfo Ramírez (coord.). Desafíos de la educación media superior (pp. 161-217). México: Senado de la República/ Instituto Belisario Domínguez.

Reguillo, Rossana (2000). Emergencia de culturas juveniles. Estrategias de desencanto. Bogotá, Colombia: Grupo Editorial Norma. 
Rockwell, Elsie (1987). Reflexiones sobre el proceso etnográfico, 1922-1985. México, DF: Departamento de Investigaciones Educativas.

Saccone, Mercedes (2016). La acreditación de asignaturas en la vida cotidiana escolar. Un estudio etnográfico en el contexto de la obligatoriedad de la educación media superior. Tesis de maestría. DIE-Cinvestav, México.

Saccone, Mercedes y Weiss, Eduardo (2017). La acreditación de asignaturas en la vida cotidiana escolar. Un estudio etnográfico en el contexto de la obligatoriedad de la educación media superior. Propuesta Educativa, vol. 1, núm. 47, pp. 119-128. Recuperado de http://www.redalyc.org/articulo. oa?id=403052805013

Secretaría de Educación Pública-Direcccion General de Bachillerato (SEP-DGB) (2014). Mapa curricular del TBC. Recuperado de http://www.dgb.sep.gob. $\mathrm{mx} /$ servicios-educativos/telebachillerato/normatividad/Mapa_CURRICULAR_tbc_2014.pdf

SEP-COPEMS (2012). Reporte de la Encuesta Nacional de Deserción en la Educación Media Superior. Recuperado de http://www.sems.gob.mx/work/models/sems/Resource/10787/1/images/Anexo_6Reporte_de_la_ENDEMS.pdf

Sergio, Germán (2015). Educación media superior: oferta actual y desafíos para la universalización de su cobertura. En Rodolfo Ramírez (coord.). Desafíos de la educación media superior (pp.15-61). México: Senado de la República/Instituto Belisario Domínguez.

Silva, Hugo (2016). El abandono escolar de los Jóvenes del Bachillerato Tecnológico Agropecuario. Un estudio cualitativo. Publicación libre. México.

Simmel, George (2002). Sobre la individualidad y las formas sociales. Escritos escogidos. Buenos Aires, Argentina: Universidad Nacional de Quilmes.

SITEAL (2015). Escolarización en América Latina 2000-2013. Resumen estadístico comentado. Recuperado de http://www.siteal.org/sites/default/files/ rec_siteal_2_2015_04_28.pdf

Székely, M. (2015). Estudio sobre los principales resultados y recomendaciones de la investigación y evaluación educativa en el eje de prevención y atención a la deserción escolar en educación media superior. Informe final. México.

Tapia, Guillermo (2015). Estudiantes en la transición rural-urbana del Bajío. Los significados del bachillerato y del trabajo. Tesis de doctorado, DIE-Cinvestav, México.

Weiss, Eduardo (2017a). Hermenéutica y descripción densa versus teoría fundamentada. Revista Mexicana de Investigación Educativa. vol. XXII, núm. 73, pp. 637-654. Recuperado de http://www.scielo.org.mx/pdf/rmie/ v22n73/1405-6666-rmie-22-73-00637.pdf

Weiss, Eduardo (2017b). El telebachillerato comunitario. Una innovación curricular a discusión. Revista Latinoamericana de Estudios Educativos, vol. XLVII, núms. 3 y 4, pp. 7-26. Recuperado de http://cee.edu.mx/home/la-agendaeducativa-3/

Weiss, Eduardo (2015a). El abandono escolar en la educación media superior: dimensiones, causas y políticas para abatirlo. En Rodolfo Ramírez (coord.). Desafíos de la educación media superior (pp. 81-159). México: Senado de la República/Instituto Belisario Domínguez.

Weiss, Eduardo (2015b). Más allá de la socialización y de la sociabilidad: jóvenes y bachillerato en México. Revista Educação e pesquisa, vol. 41, núm. especial, pp. 1257-1272. Recuperado de http://www.scielo.br/pdf/ep/ v41nspe/1517-9702-ep-41-spe-1257.pdf 
Weiss, Eduardo (coord.) (2012). Jóvenes y bachillerato. México: ANUIES.

Weiss, Eduardo (2006). Diagnóstico de las prácticas y procesos curriculares en los bachilleratos integrales comunitarios. Informe de evaluación solicitada por la Coordinación General de Educación Intercultural Bilingüe, SEP, México.

Weiss, E., Guerra, I., Guerrero, E., Hernández, J., Grijalva, O. y Ávalos, J. (2009, noviembre). Jóvenes y bachillerato en México: el proceso de subjetivación, el encuentro con los otros y la reflexividad. Propuesta Educativa, año 18, núm. 32, pp. 85-104. 